Chirurg 2013 $\cdot 84: 907$

DOI 10.1007/s00104-013-2613-x

Online publiziert: 27. September 2013

c) Springer-Verlag Berlin Heidelberg 2013

O. Strobel · M.W. Büchler

Klinik für Allgemein-, Viszeral- und Transplantationschirurgie, Universität Heidelberg

\title{
Rekonstruktion nach partieller Pankreatoduodenektomie
}

\section{Pankreatojejunostomie oder Pankreatogastrostomie?}

\section{Originalpublikation}

Topal B, Fieuws S, Aerts R et al (2013) Pancreaticojejunostomy versus pancreaticogastrostomy reconstruction after pancreaticoduodenectomy for pancreatic or periampullary tumours: a multicentre randomised trial. Lancet Oncol 14:655-662

\section{Hintergrund und Fragestellung}

Die Pankreasfistel ist die häufigste schwerwiegende Komplikation nach partieller Pankreatoduodenektomie. Das beste Rekonstruktionsverfahren zur Vermeidung von Pankreasfisteln ist daher von zentralem Interesse. Hierfür stehen die Pankreatojejunostomie (PJ) und die Pankreatogastrostomie (PG) zur Verfügung. In randomisierten Studien und Metaanalysen zum Vergleich beider Rekonstruktionsverfahren konnte bisher kein relevanter Unterschied bezüglich der Fistelraten nachgewiesen werden. Topal et al. führten in Belgien eine neue multizentrische randomisiert kontrollierte Studie zum Vergleich von PJ und PG durch.

\section{Methoden}

Von 2009 bis 2012 wurden 329 Patienten nach Resektion von Pankreas- und periampullären Tumoren anhand des Gangdurchmessers stratifiziert ( $\leq 3 \mathrm{~mm}$ vs. $>3 \mathrm{~mm}$ ) und intraoperativ in die Gruppen $\mathrm{PJ}(\mathrm{n}=167)$ und PG $(\mathrm{n}=162)$ randomisiert. Die Operateure mussten mindestens jeweils 5 PJ und PG durchgeführt haben. Anastomosierungstechnik (ein- oder zweireihig, Einzelknopf oder fortlaufend) und Nahtmaterial waren nicht standardisiert. Sandostatin wurde intraoperativ und bis 7 Tage postoperativ verabreicht.
Primärer Endpunkt war die Inzidenz klinisch relevanter Pankreasfisteln (Grad B/C, International Study Group on Pancreatic Fistula -Definition) bis 2 Monate postoperativ.

\section{Ergebnisse}

B/C-Pankreasfisteln traten bei $14 \%$ der Patienten auf und waren nach PJ signifikant häufiger als nach PG (PJ: 19,8\% vs. PG: $8,0 \% ; p=0,002)$. Die Gesamtrate an Pankreasfisteln (inklusive biochemischer Fisteln) lag nach PJ bei 31,1\% und nach PG bei $20,4 \%$. Bei Patienten mit einem Gangdurchmesser $\leq 3 \mathrm{~mm}$ und eher weichem Parenchym traten B/C-Fisteln nach $\mathrm{PJ}$ in $24,5 \%$ und nach PG in 10,2\% auf, bei Patienten mit Gangdurchmesser $>3$ mm und härterem Parenchym (niedriges Risiko) nach PJ in $12,3 \%$ und nach PG in $4,7 \%$. Eine verzögerte Magenentleerung war nach PJ weniger häufig als nach PG (8 vs. $15 \%)$. Bezüglich anderer Komplikationen, Krankenhausmortalität (PJ: 5\% und PG: 3\%) und Liegedauer (PJ: 18 Tage vs. PG: 19 Tage) gab es keine Unterschiede.

\section{Diskussion}

Die Autoren folgern, dass die Pankreatogastrostomie als sichereres Rekonstruktionsverfahren bevorzugt werden sollte. Wir glauben, dass die Studie mehrere Mängel hat, die die Interpretation und Anwendbarkeit der Ergebnisse stark einschränken:

- Die routinemäßige Gabe von Sandostatin und Liegedauern um 18 Tage spiegeln nicht die aktuelle Praxis in der Pankreaschirurgie wider.

- Die fehlende Standardisierung der Anastomosierungstechniken erschwert eine Vergleichbarkeit durch Addition zusätzlicher Variablen.

- Die Fistelraten nach PJ (klinisch relevante Fisteln in 20\%) sind wesentlich höher als von Zentren und in anderen randomisiert-kontrollierten Studien berichtet. Die scheinbare Überlegenheit der PG ist daher keinesfalls übertragbar auf Zentren mit besseren Ergebnissen nach PJ.

- Ein Grund für die hohen Fistelraten könnte die Beteiligung von Chirurgen sein, die Erfahrung mit nur 5 PJ und PG hatten und damit die Lernkurve sicher nicht durchschritten hatten.

\section{Fazit}

Denkbar ist, dass die PG als einfacheres Verfahren schneller erlernt werden kann und bei kleinerer Fallzahl sicherer ist, während die Vorteile der PJ als technisch anspruchsvolleres Verfahren erst bei größerer Fallzahl zum Tragen kommen. Interessant wäre hier die Aufschlüsselung der Ergebnisse anhand der Erfahrung der Chirurgen mit dem jeweiligen Verfahren. Wir hoffen, dass die in Deutschland laufende RecoPancStudie zur weiteren Klärung des Themas beiträgt.

\section{Korrespondenzadresse}

PD Dr. O. Strobel

Klinik für Allgemein-, Viszeralund Transplantationschirurgie, Universität Heidelberg, Im Neuenheimer Feld 110, 69120 Heidelberg Oliver.Strobel@med.uni-heidelberg.de

Interessenkonflikt. O. Strobel und M.W. Büchler geben an, dass kein Interessenkonflikt besteht. 